\title{
Bridging Database Analysis with Microrheology to Reveal Super-Hydrodynamic Conductivity Scaling Regimes in Ionic Liquids
}

\author{
Ryan K. Cashen, Megan M. Donoghue, Abigail J. Schmeiser, Matthew A. Gebbie* \\ Department of Chemical and Biological Engineering, University of Wisconsin - Madison, Madison, \\ Wisconsin 53706
}

Keywords

collectivity, ion correlations, Nernst-Einstein, ion transport, electrolyte

\begin{abstract}
Ion transport through electrolytes critically impacts the performance of batteries and other electrochemical devices. Many frameworks used to predict and tune ion transport, such as the Nernst-Einstein model, assume hydrodynamic transport mechanisms, and hence focus on maximizing electrolyte conductivity by minimizing bulk viscosity. However, the emergence of solid-state electrolytes illustrates that selective, nonhydrodynamic ion transport provides promising avenues for enhancing ionic transport in electrolytes. Increasingly, selective ion transport mechanisms, such as hopping, are proposed for concentrated electrolytes, including ionic liquid-derived materials. Yet viscosity-conductivity scaling relationships in ionic liquids are still often analyzed with hydrodynamic models. Here, we report a data-centric analysis of how well hydrodynamic transport models describe the scaling between viscosity and conductivity in neat ionic liquids by merging three databases to bridge physical properties and chemical descriptors. With this expansive data set, we constrained our scaling analysis using ion sizes defined using simulated molecular volumes, as opposed to prior approaches that estimate sizes from activity coefficients or rely on ad-hoc estimates. Remarkably, we find that many commonly studied ionic liquids exhibit positive deviations from the Nernst-Einstein model, implying that ions move faster than hydrodynamic limitations should allow. We experimentally verify these positive deviations in a common class of ionic liquids using microrheology and conductivity measurements. Our results highlight overlooked super-hydrodynamic regimes in ionic liquid viscosity-conductivity scaling and point to opportunities to understand mechanisms of correlated ion motion in ionic liquids. We further show data science and machine learning tools can improve predictions of conductivity from molecular properties, including demonstrating predictions can be made using only computational features. Our findings reveal that many ionic liquids exhibit super-hydrodynamic viscosityconductivity scaling, which could be harnessed to influence the behavior of electrochemical devices.
\end{abstract}




\section{Introduction and Background}

As researchers work towards a new generation of energy dense batteries, safety and sustainability are becoming increasingly important considerations. Increasingly, liquid electrolytes with extremely high ion concentrations and solid-state electrolytes are explored to increase battery safety and efficiency. An emerging paradigm is that these materials enhance batteries by facilitating ion-selective transport, ${ }^{1-4}$ which increases the reversibility of battery charge cycles.$^{5-7}$ Further, super-concentrated and solid state electrolytes have greatly enhanced thermal and electrochemical stability relative to conventional organic electrolytes. ${ }^{8}$

Many explanations for the high performance of super-concentrated and solid-state electrolytes center around non-hydrodynamic transport mechanisms, such as "ion hopping," to explain how these materials could facilitate efficient ion motion. For example, super-concentrated electrolytes have much higher viscosity than conventional organic electrolytes due to significant ion correllations. ${ }^{5,9-11}$ Yet such materials can facilitate battery charging at rates that exceed the limits of conventional organic electrolytes, ${ }^{6}$ presumably due to a combination of enhanced stability and ion-specific transport via mechanisms that facilitate motion at rates exceeding hydrodynamic limits. Such a picture is consistent with the emergence of solid state electrolytes, where selective ion transport is key to performance. ${ }^{12}$

In most cases, nanoscale structural organization of preferential ion transport pathways are key to superhydrodynamic transport. Notably, ionic liquids exhibit pronounced nanoscale structuring, so we hypothesize that ionic liquids may also exhibit decoupling of viscosity and conductivity, even as pure materials. To evaluate this hypothesis, we performed a distinctly comprehensive viscosity-conductivity scaling analysis by drawing from the wealth of experimental and computational data available in the scientific literature to determine how well the Nernst-Einstein theory of ion transport describes ionic liquids.

Ionic liquids are comprised of bulky and often asymmetric ions which prevent packing into a crystalline lattice, making them liquids at near-ambient temperatures. ${ }^{13-15} 16$ Ionic liquids possess a unique combination of properties that can be especially advantageous for batteries, including negligible volatility, high thermal stability, low flammability, and high conductivity. ${ }^{14,17-20}$ Hence, increased understanding of transport scaling relationships in ionic liquids could lead to new frameworks for engineering ionic liquids as safe electrolytes for energy dense batteries. Though ionic liquids are not commonly used in commercial batteries, they have been heavily investigated as electrolytes for safe, next-generation batteries. ${ }^{5,8,13,20-31}$

Notably, classical hydrodynamic design relationships, such as the Nernst-Einstein and Walden frameworks, imply that ionic liquids should be poor electrolytes due to high viscosity compared to conventional carbonate blends. Nevertheless, recent studies suggest that ionic liquids can enable fast cycling batteries, suggesting that classical hydrodynamic mechanisms may not provide the full picture of dynamics in ionic liquids. At the fundamental level, systematic modulation of cation and anion structures renders ionic liquids ideal model systems to study how different chemical features influence ionic 
correlations and transport, which promise to yield new insights relevant to many areas of science and technology where ionic correlations play a major role. ${ }^{32-34}$

Here, we report a data-centric analysis of how well hydrodynamic transport models describe scaling between viscosity and conductivity in ionic liquids by merging three databases to bridge physical properties and chemical descriptors. We aim to use this broad scale approach to provide insight into why certain ionic liquids exhibit advantages for electrochemical devices. For example, ionic liquids with conductivity much lower than that predicted by viscosity may be poor battery electrolytes, while those with conductivity exceeding predictions suggest super-hydrodynamic transport mechanisms, such as ion-hopping, ${ }^{35,36}$ may be present. This interpretation has been applied to solvent-in-salt electrolytes, which may exhibit superhydrodynamic conductivities. ${ }^{8,36}$ Our aim is to investigate when super-hydrodynamic transport scaling is present in ionic liquids and how this connects to molecular structures as a step towards the rational design of self-assembled electrolytes with specific molecular level ion transport pathways.

\section{Transport Scaling Relationships in Ionic Liquids}

One of the most widely used hydrodynamic electrolyte viscosity-conductivity scaling relationships for ionic liquids is the Walden framework, where molar conductivity $\left[\mathrm{Sm}^{2} / \mathrm{mol}\right]$ is plotted against the inverse viscosity $\left[(\mathrm{Pa} * s)^{-1}\right]$ and an empirical temperature-dependent constant $\left[\mathrm{C}^{2} /(m * m o l)\right]$ is calculated to correct model deviations.

$$
\Lambda_{\text {pred }}=\frac{\mathrm{k}(\mathrm{T})}{\eta}
$$

In the Walden approach, ionic liquids are compared to dilute $\mathrm{KCl}$ solutions since $\mathrm{KCl}$ is assumed to benchmark the hydrodynamic behavior of fully dissociated strong, electrolytes. For many ionic liquids, the molar conductivities predicted from hydrodynamic scaling theory fall below that of the dilute $\mathrm{KCl}$ solution predicted using the same frameworks even after accounting for differences in viscosity. Traditionally, these sub-hydrodynamic deviations are interpreted as an indication that ionic liquids are either weakly dissociated or exhibit transport processes that are influenced by differing temperature dependencies of ion motion and structural relaxation, as has been observed in glassy and crystalline salts. ${ }^{37-39}$

Notably, hydrodynamically-decoupled mechanisms of ion motion, such as structural-diffusion transport and ion hopping, are permitted within the Walden framework. Electrolytes with "super hydrodynamic" ion transport are often identified by falling above a $\mathrm{KCl}$ reference line, meaning the measured conductivity is larger than would be expected if transport was limited by hydrodynamic drag. Yet, the Walden framework is empirical, and deviations from Walden ideality do not define transport mechanisms in electrolytes. ${ }^{36,40,41}$

In contrast, conductivity models that are based in fundamental mechanistic theories provide quantitative frameworks to benchmark the presence of super-hydrodynamic behavior that may be beneficial for battery 
electrolytes. For example, the widely used Nernst-Einstein model enables prediction of molar conductivity without requiring addition of empirical parameters. This model assumes independent charge mobility such that directly summing the diffusive terms can predict a molar conductivity in the form of:

$$
\Lambda_{\text {pred }}=\frac{\mathrm{N}_{\mathrm{A}} \mathrm{e}^{2}}{\mathrm{kT}}\left(v_{+} \mathrm{z}_{+}^{2} \mathrm{D}_{+}+v_{\mathrm{z}_{-}}^{2} \mathrm{D}_{-}\right)
$$

Here, $N_{A}$ is Avogadro's number [ $\left.\mathrm{mol}^{-1}\right], e$ is the electronic charge $\left[1.602 * 10^{-19} \mathrm{C}\right], k$ is Boltzmann's constant $[J / K], T$ is the absolute temperature $[K], v$ is the stoichiometric coefficient of the ion dissociation process for each ion, $z$ is the ionic charge, and $D$ is the diffusion coefficient of each ion $\left[\mathrm{m}^{2} / \mathrm{s}\right]$. Further, if the sizes of charge carriers are known, the Stokes-Einstein diffusion coefficient can be substituted to calculate a hydrodynamic prediction of the molar conductivity based on viscous drag:

$$
\Lambda_{\text {pred }}=\frac{\mathrm{N}_{\mathrm{A}} \mathrm{e}^{2}}{6 \pi \eta}\left(\frac{\nu_{+} \mathrm{z}_{+}^{2}}{\mathrm{r}_{+}}+\frac{\nu_{-} \mathrm{z}_{-}^{2}}{\mathrm{r}_{-}}\right)
$$

Where $r_{i}[m]$ is the radius of a charge carrier moving through the fluid. Most ionic liquids are monovalent, canceling the stoichiometric and ionic charge terms. The Nernst-Einstein model, in this form, assumes that ion transport can be characterized by continuum hydrodynamics and that cation and anion mobility are independent. While ionic liquids do not rigorously meet these assumptions, the Nernst-Einstein model can predict conductivity scaling for some ionic liquids. ${ }^{42-44}$ However, many ionic liquids strongly deviate from Nernst-Einstein predictions.

In this work, we use deviations from the Nernst-Einstein model to screen for non-classical transport mechanisms in ionic liquids. A key innovation of our work is to constrain Nernst-Einstein predictions using physical estimates of ion sizes determined by the calculated volume of individual ions from molecular modeling. This differs from prior approaches, where ion sizes may be fitting parameters determined from activity models, geometric estimates, or other approaches. ${ }^{43-46}$

Previously, deviation from the predicted conductivity in the Nernst-Einstein expression has often been interpreted as "ionicity" or "dissociation", the fraction of free ions contributing to the conductivity of the sample. ${ }^{43,44,46}$ This deviation is expressed as the Nernst-Einstein ratio

$$
\mathrm{I}=\frac{\Lambda_{\text {obs }}}{\Lambda_{\text {pred }}}
$$

the ratio of the observed alternating current molar conductivity to the predicted molar conductivity. Prior interpretations of this ratio as the fractional dissociation requires that all ionic liquids should have an ionicity ratio less than one. Here, we refer to this ratio as the "Nernst-Einstein ratio" without adopting the interpretation as a fraction of free ions. Significantly, only four parameters are required to quantify the 
Nernst-Einstein ratio: viscosity, AC conductivity, density, and ion sizes. We aim to evaluate how deviations in this Nernst-Einstein ratio can inform understanding of transport in concentrated electrolytes.

\section{Data Centric Approach to Scaling Analysis}

To enable a new approach for evaluating how hydrodynamic transport scaling theories apply to ionic liquids, we developed a database tool to link the NIST repository of ionic liquid physical properties - IL Thermo $^{47,48}$ - with the extensive PubChem ${ }^{49,50}$ and RDKit ${ }^{51}$ databases of calculated ion descriptors. Notably, this enabled us to perform the first extensive scaling analysis of viscosity-conductivity relationships in over 250 ionic liquids and 2700 data points from 116 different publications, after data cleaning. Critically, our analysis draws exclusively on computationally derived ion size estimates to evaluate Nernst-Einstein conductivity predictions and compare them to experimental measurements. Prior transport scaling analyses often use a combination of activity models, ad-hoc estimates of ion sizes from van der Waals dimensions or comprised limited numbers of ionic liquids to study.

One of our most important findings is that many ionic liquids exhibit super-hydrodynamic deviations from the Nernst-Einstein theory, indicated by molar conductivities exceeding Nernst-Einstein predictions.

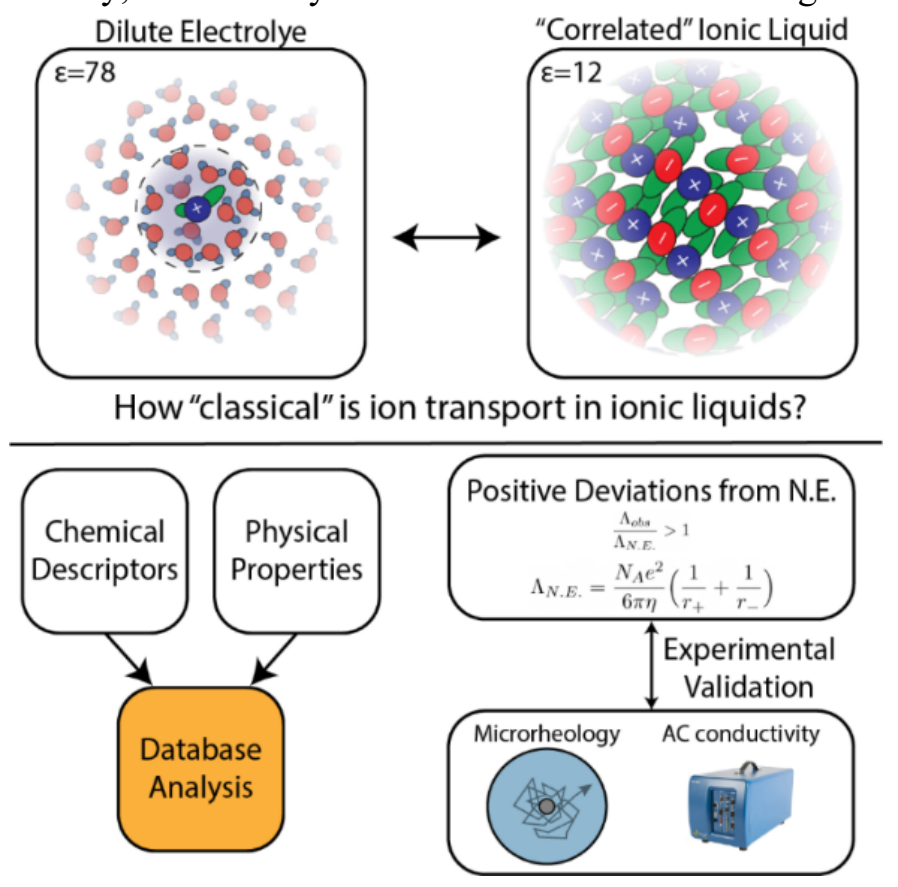

Figure 1. We quantify how classical viscosity-conductivity scaling frameworks, such as the NernstEinstein model and Walden frameworks, apply to ionic liquids using a database that merges chemical descriptors and physical properties from public sources: PubChem, RDKit, and ILThermo. Critically, we constrained ion sizes using calculated volumes of individual ions to physically constrain NernstEinstein predictions. We find many ionic liquids exhibit molar conductivities larger than classical hydrodynamic predictions, as evidenced by a "Nernst-Einstein ratio" greater than 1 . We confirmed this finding experimentally for a common class of imidazolium ionic liquid. Our findings and analysis show that super-hydrodynamic conductivity mechanisms may be common in ionic liquids. 
We interpret deviations from Nernst-Einstein predictions to originate from correlated ion motion, which is a mechanism that could consistently apply to both super-hydrodynamic (greater than 1) and subhydrodynamic (less than 1) "Nernst-Einstein ratios." This stands in contrast to the "ionicity" interpretation which, by definition, requires the molar Nernst-Einstein ratio to be less than one.

Further, the extensive data available in our database enables us to go beyond analysis of Nernst-Einstein deviations to predict the conductivity of ionic liquids using computational and experimental properties to identify features that are most important in determining conductivity. Despite significant deviations from the Nernst-Einstein model, we find that Stokes-Einstein diffusivities of cations and anions are still the two most important parameters for predicting the molar conductivity. Accounting for geometric molecular features of ions, such as eccentricity, can modestly improve predictions. Finally, using a neural network, we demonstrate that ionic liquid molar conductivity can be estimated using only computationally derived features, which suggests data science tools can enable rapid screening of molar conductivity of ionic liquids.

\section{Methods}

To calculate predicted Nernst-Einstein conductivities, we use data from reported viscosity measurements and estimate ion radii from the ion-volume given by the 3D conformation given from the PubChem database. ${ }^{49,50}$ Using computationally derived sizes of individual ions enables the broadest possible analysis of the Nernst-Einstein model in ionic liquids, and importantly, provides a constrained analysis of hydrodynamic conductivity predictions. We estimate ionic radii from the radius of a sphere containing the equivalent volume that is occupied by the ion's molecular structure upon relaxation using molecular dynamics simulations using databases available from PubChem. ${ }^{50}$

We also highlight that our approach of constraining ion sizes to physical dimensions provides a lowerlimit to the size of charge carriers, since solvation is not included, thereby yielding a conservative lower bound estimate of the Nernst-Einstein ratio (Eq. 4). This choice of ion size directly predicts ionic diffusivity, and therefore conductivity, if the mechanism of transport is dictated by hydrodynamics of a spherical charge carrier moving in a viscous liquid. Observed molar conductivities are then calculated by normalizing reported specific conductivity measurements by the overall concentration of the ionic liquid via the density and molecular weight of the ionic liquid, as is done in prior works. ${ }^{43,44,52,53}$

To validate database findings, we perform alternating current (AC) conductivity measurements and particle tracking microrheology to measure viscosity in a class of common ionic liquids, 1-alkyl-3methylimidazolium bis(trifluoromethylsulfonyl)imide ( $\mathrm{C}_{\mathrm{n}} \mathrm{MIm}$ TFSI, $\left.\mathrm{n}=2,4,10,12\right)$. Microrheology enables viscosity to be measured precisely in small samples, making it an ideal approach using small amounts of fluid in an isolated environment. ${ }^{54}$ Microrheology can have major advantages for studying ionic liquids, ${ }^{55,56}$ as it provides the ability to quantify Brownian Dynamics across several orders of magnitude in 
time (1 $\mu s-10$ s or longer) while preserving inert measurement environments. Detailed information on microrheology and conductivity measurements and analysis is reported in the "Experimental" section below. Density measurements were selected from the ILThermo database, using widely accepted values between various publications. ${ }^{47,48}$

In addition to exploring the applicability of hydrodynamic electrolyte design relationships in ionic liquids, our approach enables us to explore hypotheses about which features drive conductivity changes, as well as if a better prediction of ionic liquid conductivity can be achieved using experimental and chemical descriptors. For these tasks, we employed a variety of regression techniques, including Least Absolute Shrinkage and Selection Operator (LASSO), decision-tree, random-forest, and artificial neural network regressions for feature selection and conductivity prediction using varying degrees of model complexity, and therefore, interpretability. ${ }^{57} \mathrm{We}$ split selected features into computational and experimental categories. Feature selection for determining importance is performed using all scaled features, whereas robust predictions using complex models, such as neural networks, were performed using only computational predictors to assess the ability to screen ionic liquids using only computationally derived features from PubChem and RDKit. Regressions are made after scaling features to zero mean and unit variance.

\section{Results}

\section{Nernst-Einstein Deviations}

Our database approach enables us to determine the Nernst-Einstein molar conductivity prediction based on viscosity and ion sizes for each ionic liquid and compare it to the measured molar conductivity from electrochemical measurements. This relationship is shown in Figure 2. A wide variety of deviations from the Nernst-Einstein model are exhibited. For simplicity of reference, we use three quantiles to place ionic liquids into bins using the magnitude of Nernst-Einstein deviations. These categories can be viewed as low, moderate, and high slope, meaning high Nernst-Einstein ratio, ionic liquids. A key finding of our research is that a significant number of ionic liquids demonstrate higher conductivities than the Nernst-Einstein predicted conductivity, implying super-hydrodynamic ion transport as discussed in the following section. Again, we highlight that our choice of ion size is a conservative underestimate of the charge carrier size, such that our Nernst-Einstein ratios are also conservatively low estimates. This is an important consideration given our novel finding that many ionic liquids demonstrate conductivity that surpasses hydrodynamic expectations $(I>1)$ when applying the Nernst-Einstein formulation. We similarly see observed molar conductivities larger than predicted when showing the Walden relationship applied to ionic liquids in Figure S3, which demonstrates ionic liquids falling above, near, and below the reference line. 


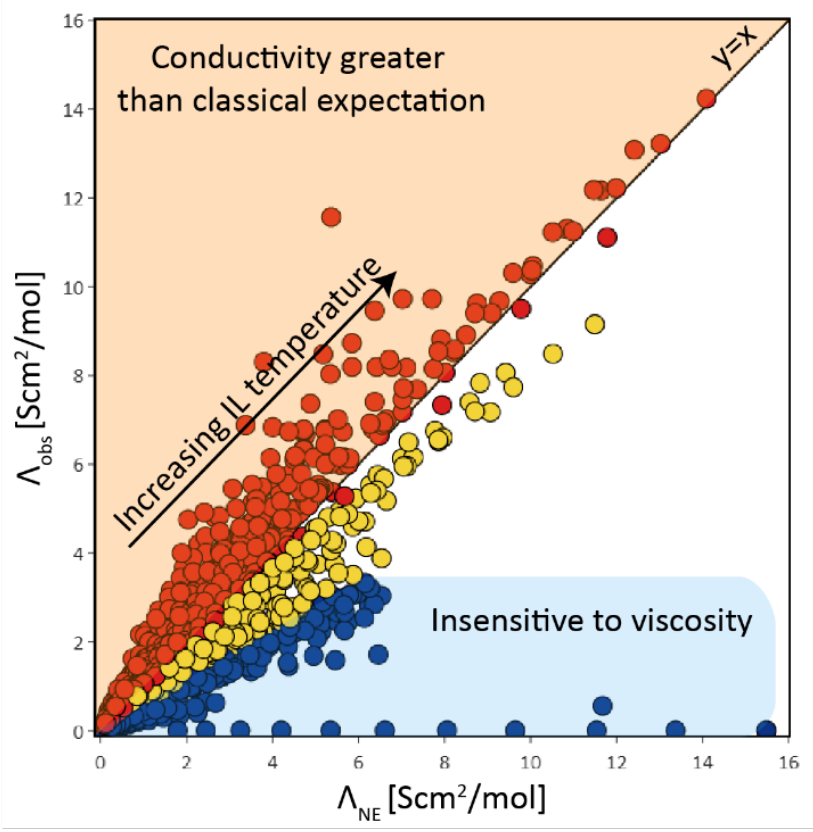

Figure 2. Calculation of Nernst-Einstein conductivity using molecular ion size estimates. Various behaviors are demonstrated in ionic liquids, including positive deviations, slight negative deviations, and significant negative deviations. For each ionic liquid, properties are measured at a range of temperatures to yield a line for each ionic liquid. Red represents ionic liquids with the largest conductivities relative to the Nernst-Einstein conductivity, blue represents significant negative deviations from Nernst Einstein, and yellow represents those below, but near, Nernst-Einstein hydrodynamic predictions. Thus, many ionic liquids appear to demonstrate super-hydrodynamic transport, as charge mobility is not limited by classic hydrodynamic-based transport models.

In addition to ionic liquids that display super-hydrodynamic transport scaling, Fig. 2 shows several ionic liquids where conductivity is largely insensitive to viscosity. These materials are comprised of anions with oxygenated polar end groups, such as carboxylates and sulfates, as well as larger cations, including variants of the imidazolium cation with the $\mathrm{N} 1-\mathrm{CH}_{3}$ group replaced with an $\mathrm{N}-\mathrm{H}$ bond. These commonalities suggest that hydrogen bonding plays a strong role in dictating how viscosity modulates ion mobility. An interactive plot of Fig. 2 with components and values shown while hovering over data values is available as a supplementary file titled "Interactive Nernst-Einstein Plot.html". Additionally, images of the ions in ionic liquids with low, medium, and high Nernst-Einstein slopes are included as supplementary files.

Next, we performed coupled conductivity and viscosity measurements to validate the finding of positive deviations from the Nernst-Einstein models in common ionic liquids. Using coupled microrheology and $\mathrm{AC}$ conductivity in the class of 1-alkyl-3-methylimidazolium bis(trifluoromethylsulfonyl)imide $\left(\mathrm{C}_{\mathrm{n}} \mathrm{MIm}\right.$ TFSI) imidazolium ionic liquids, we find deviations from Nernst-Einstein conductivity models are similar in magnitude to that observed in our database analysis, and that deviations decrease as alkyl chain lengths increase (Fig. 3). Additional experimental details are presented in the experimental section below. Importantly our analysis uses the ion size estimated without considering bound solvation shells, making 
our predicted conductivity larger at the same measured conductivity, resulting in the lowest estimate of the Nernst-Einstein ratio. Even with this conservative estimate, we consistently observe conductivities higher than hydrodynamic limitations should allow.

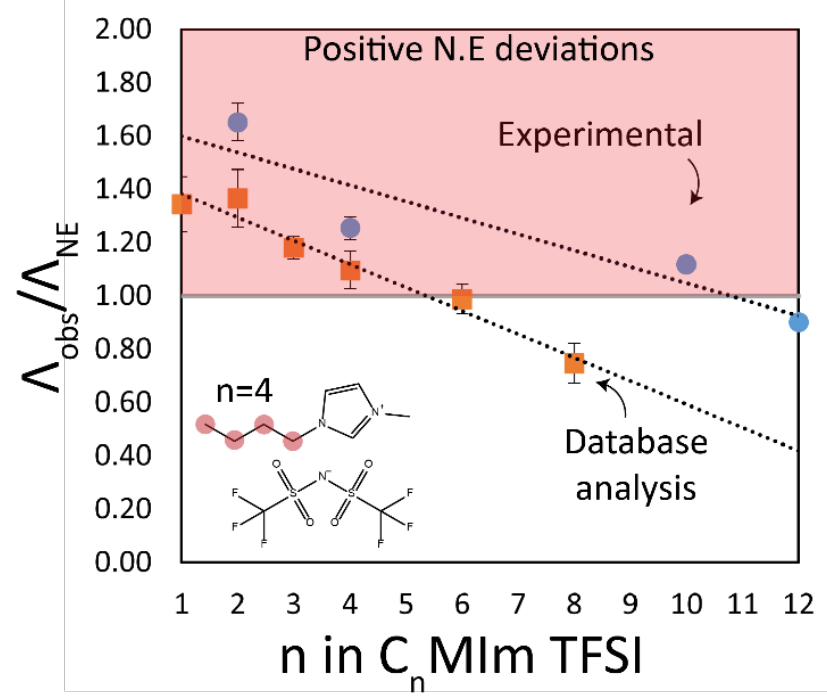

Figure 3. Ratio of observed to Nernst-Einstein (NE) predicted molar conductivity as a function of the alkyl chain length in $\mathrm{C}_{\mathrm{n}}$ MIm TFSI as determined from our database analysis and experimental microrheology and conductivity measurements. Positive deviations form Nernst-Einstein are observed but are found to decrease with increasing alkyl chain length. Database error bars represent propagated uncertainty reported from error values of each relevant property in the database. Experimental error bars represent a 95\% confidence interval of the measured or reported values.

The Nernst-Einstein ratio can also be calculated for each ionic liquid at each temperature to gain physical insight about possible origins of deviations in viscosity-conductivity scaling. The temperature dependence of the Nernst-Einstein ratio is shown in Figure 4. We see that in nearly all cases, the Nernst-Einstein ratio decreases as a function of the temperature, though some ionic liquids show weak temperature dependence. We elaborate on the importance of this finding in the discussion section. 


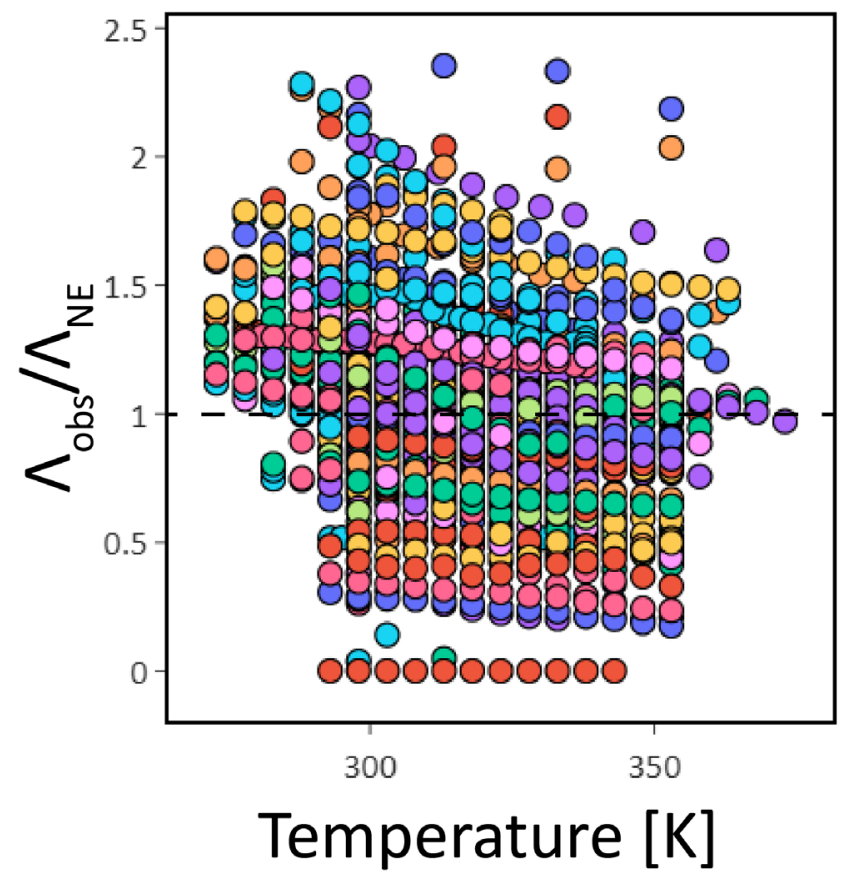

Figure 4. Temperature dependence of the ratio of the measured and Nernst-Einstein predicted molar Nernst-Einstein ratio, i.e. the Nernst-Einstein ratio, as a function of the temperature. In nearly all cases, the Nernst-Einstein ratio decreases in temperature. This finding suggests that the NernstEinstein ratio is not indicative of ionic liquid dissociation since dissociation is expected to increase with temperature via the Boltzmann population distribution (Eq. 5). Colors represent unique ionic liquids, though colors may be repeated due to limitations in color choice.

\section{Conductivity Predictions}

Expanding upon the information available in our merged database, we employed a variety of regression techniques, including LASSO, random-forest, and neural net regressors to gain further insight into how well ionic liquid conductivity can be predicted using a variety of available descriptors. Our regression implementation, including preprocessing, hyperparameter tuning, cross validation performance, etc., is discussed in the SI, sections S4-S5.

Our first approach included LASSO regression with computational and experimental features in the database to select features that are most important for prediction of molar conductivity. Restricting the model to select only two features, we find that the Stokes-Einstein diffusion coefficients of the cation and anion, from experimental viscosity and computational ion sizes, are the two most important features. Performing 5-fold cross-validation to select the number of features that yields the best regression results in an interpretable list of features that contribute to increasing or decreasing the conductivity. The table of weighted feature coefficients and the model performance are provided in section S4 of the SI. Looking at the parameters that further improve Stokes-Einstein-based conductivity predictions, we note that smaller flexible symmetric anions and less flexible cations generally lead to increased molar conductivity. 
An interpretable decision tree model, with all features included, supports these findings. We see that high molar conductivity values are associated with high cation Stokes Einstein diffusivity, high anion Stokes Einstein diffusivity, small cations, low viscosity, and cations without significantly low eccentricity, cations whose characteristic length scales are nearly the same in all dimensions. Similarly, low conductivities are associated with low Stokes Einstein diffusivities, high cation self-overlap, lower-than-average rotatable bonds in the anion, and high viscosity. (Fig S5, flowchart included in supplementary files).

To evaluate if conductivity predictions can be improved, we performed random forest regressions and artificial neural network regressions. The random forest regression served as a benchmark for model performance for the neural network when both computational and experimental features are used for model training. The neural network regressor performs modestly better than the random forest regressor when all features are used for each case. Details of each model are available in section S6.

Artificial neural networks show good performance when using both computational and experimental features $\left(\mathrm{R}^{2}=0.824\right.$, Fig. S7). Intriguingly, using only computational features, we see suitable performance $\left(\mathrm{R}^{2}=0.753\right.$, Fig. 5$)$, with better predictive capability compared to the random forest model. This key finding demonstrates that computational features from PubChem and RDKit may be used for screening of molar conductivity when no experimental data is available. However, the neural network model is challenging to

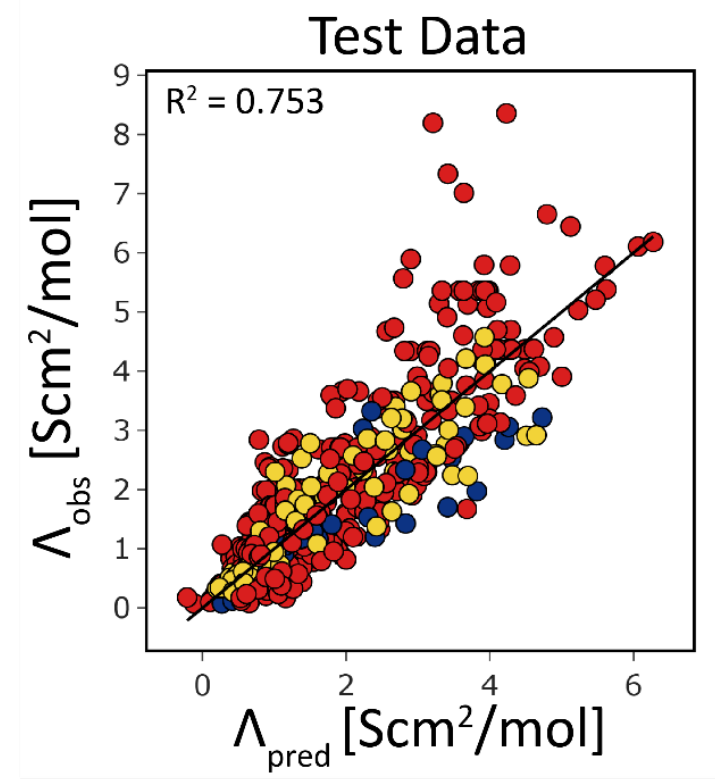

Figure 5. Neural network conductivity prediction, trained using only computational features, applied to holdout data. $20 \%$ of unique ionic liquids with required features were used to test the model. Model performance is suitable, suggesting computational features from PubChem and RDKit can be used to approximate the molar conductivity of an ionic liquid with no experimental data. Training performance, hyperparameter tuning processes, and pipeline for regression are included in section S6. Training data performance and the neural network architecture are shown in the SI Figure S8. Colors are consistent with the quantile binning, as shown in Figure 2. 
interpret, making it difficult to provide direct insight into what physical origin gives rise to deviations from the Nernst-Einstein molar conductivity.

\section{Discussion}

Walden and Nernst-Einstein plots have been used to suggest that ionic liquids should always have lower conductivities than their viscosities imply. ${ }^{4,6-8}$ This implication is, in part, based on publications which have expanded understanding of how features such as ion-polarity, concentration, temperature, and other features influence the deviation of ionic liquids and ionic liquid solutions from conductivity-viscosity models, where measured molar conductivities are often thought to be lower than predicted conductivities. Deviation from these conductivity-viscosity scaling relationships in ionic liquids are commonly rationalized from the perspective of the ionicity framework.

In the ionicity framework, deviations are typically explained as indicating that ionic liquids exhibit fractional ionic "dissociation" of around $50-80 \%$ of their Nernst-Einstein limit, suggesting that the NernstEinstein ratio is a direct measurement of the fraction of ions free to transport charge. ${ }^{43,46,52,58}$ In contrast, our constrained Nernst-Einstein model shows many ionic liquids have molar conductivities larger than hydrodynamic predictions (Fig. 2-4). Within the ionicity framework, the finding that Nernst-Einstein ratios frequently exceed one would imply greater than $100 \%$ ion dissociation in ionic liquids. Hence, the key observation that Nernst-Einstein ratios routinely exceed unity conflicts with the interpretation that the Nernst-Einstein ratio quantifies fractional ion dissociation. Indeed, molecular dynamics simulations suggest that ionic liquids are $15-25 \%$ dissociated, ${ }^{10}$ and surface forces measurements suggest even smaller effective dissociation values, below $0.1 \% .^{59,60}$

The choice of ion size can have a large influence on whether calculated Nernst-Einstein ratios exhibit deviations from ideality. Ion sizes must be defined when using the Stokes-Einstein diffusivity in the NernstEinstein model, though definitions of the ion size used can vary widely. The Stokes, or hydrodynamic, radius of the ion is often used to model the diffusing charge carrier size since counterions, or solvent species are expected to diffuse with the ionic species to some degree. Prior scaling theory analysis of ionic liquids estimated ion radii with methods including activity models ${ }^{43}$, geometric approximations, ${ }^{44,45}$ commercially available software packages, ${ }^{61,62}$ and calculation via equating diffusion coefficient measurements to the Stokes-Einstein diffusion coefficient and solving for $r .{ }^{46}$ These approaches have advanced discussion of the ionicity framework and provided useful interpretations about the conductivity of ionic liquids.

However, there is no present unifying theory about the nature of solvation or activity in ionic liquids and other concentrated electrolytes, thereby making estimates of a hydrodynamic radius challenging. Thus, modelling ion size presents a roadblock to rapid electrolyte screening using computational tools. ${ }^{63}$ Our PubChem ion size choice provides the benefit of constraining the model with estimates of ion size based 
on molecular structures. Notably, this ion size choice approximates the smallest possible size of an ionic liquid charge carrier, making it a conservative, low-end estimate of the Nernst-Einstein ratio, which is an important consideration when demonstrating the finding that the ratios are unexpectedly greater than one.

The temperature dependence of the Nernst-Einstein ratio provides additional insights into the origins of deviations from hydrodynamic viscosity-conductivity scaling in ionic liquids. Invoking the Boltzmann distribution to describe the relative population of thermally excited ions $\left[n^{*}\right]$ relative to ions participating in collective association $\left[n_{0}\right]$, it is expected that increased temperature leads to dissociation of aggregates since interaction energies become less significant compared to thermal energy. In a Boltzmann distribution, the fraction of thermally excited charge carriers is given by:

$$
\frac{\mathrm{n}^{*}}{\mathrm{n}_{0}}=\mathrm{e}^{-\mathrm{E}_{\mathrm{D}} / k \mathrm{~T}}
$$

where $E_{D}[J]$ is an energy change related to ion dissociation, which is dependent on ion interaction energies. Accordingly, classical thermodynamics requires that increasing temperatures should show an increase in the fractional dissociation, plateauing to a value of one at high temperatures. However, Figure 4 shows that in nearly all cases, the Nernst-Einstein ratio decreases as the temperature increases. This result is consistent with recent molecular dynamics studies ${ }^{10}$ and other approaches of estimating the Nernst-Einstein ratio using activity models or nuclear magnetic resonance. ${ }^{43,46}$ Hence, we conclude that the Nernst-Einstein ratio is not linked directly to the fractional dissociation as quantitatively linking the two features would suggest that increasing temperatures would increase ion aggregation.

One possible explanation for the surprising temperature dependence of the Nernst-Einstein ratio presented in Figure 4 is a pronounced decoupling of the conductivity and viscosity arising from ioncorrelations and non-classical ion-transport mechanisms, as discussed in prior works. ${ }^{4,64}$ This agrees with a developing body of work that suggests alternative meanings to the free ion fraction interpretation based on ionic correlations, the presence of charge transfer events, or proton transfer in protic ionic liquids, ${ }^{52,65-68}$ which may contribute to different temperature dependencies of conductivity and viscosity.

Taken together, we interpret that ionic liquid transport scaling is not quantitatively linked to fractional dissociation. Our database analysis supports earlier suggestions that deviations from Nernst-Einstein are best explained by ionic correlations, ${ }^{52,65,67,69}$ which can be accounted for using diffusivities determined via velocity correlation coefficients. ${ }^{35,67-71}$ Since individual ion trajectories are required, this approach is limited to molecular dynamics studies, though the concept translates to experimental studies since some superconcentrated electrolytes deviate from the Nernst-Einstein model, as the model does not account for ionic correlations. Accordingly, deviations may indicate differences in ionic liquid transport mechanisms.

Supporting this interpretation, Angell and colleagues classified a small number of materials observed to fall above the "ideal Walden line" as "super-ionic." Other electrolytes that lay above this line are known, 
including ionic liquids. ${ }^{64,72-75}$ This type of conductivity-viscosity decoupling is suggested to arise from a "special mechanism for conductance... (as for superionics)", providing a heuristic that may help identify materials that exhibit super-hydrodynamic transport. ${ }^{64}$ For example, recent studies highlight the existence of "hopping" or other directed transport processes in electrolytes with ionic correlations or significant ionic nanostructure suggest hydrodynamically-decoupled, super-hydrodynamic modes of ion transport. ${ }^{28,36,76-80}$

However, the Walden model is empirical, so evidence of super-hydrodynamic ion-transport is more so based on quantitative similarity to other materials such as ionic glasses than direct evaluation of a proposed transport mechanism. In contrast, the Nernst-Einstein model makes specific assumptions about hydrodynamic transport, enabling us to ask when and how ion motion in ionic liquids neat ionic liquids differ from predictions of classical hydrodynamic theory. Given the findings shown in Figures 2-4, we conclude that many ionic liquids exhibit super-hydrodynamic scaling, which may be related to non-classical mechanisms of ion transport.

Recent publications show hints of positive deviations from the Nernst-Einstein model in ionic liquids, but these findings are typically subtle, limited to very few ionic liquids, and the findings are often suggested to be error. ${ }^{43,74}$ However, Nernst-Einstein ratios greater than one are common in solid electrolytes, since transport is super-hydrodynamic. ${ }^{71}$ Our findings suggest this phenomena is also characteristic of many more ionic liquids than previously appreciated, pointing towards exciting opportunities for tuning ion transport in super-concentrated electrolytes. Translating super-hydrodynamic ion transport frameworks to blends for battery applications, like Li salt/ionic liquid blends, remains a promising area for demonstrating the importance of decoupling conductivity and viscosity by tuning ion transport mechanisms. ${ }^{35,36,79,80}$

\section{Conclusion}

These findings, along with previous works, demonstrate the intriguing possibility of super-hydrodynamic motion of ionic liquids. Of particular interest to energy storage applications is if the enhanced motion of ionic liquid dopants, like lithium salts, can achieve super-hydrodynamic transport of specific ions of interest to provide benefits of ion transport selectivity. ${ }^{35}$ Our broad-spectrum analysis of conductivity-viscosity scaling in ionic liquids using both experimental measurements and molecular descriptors has enabled us to reveal ionic liquids can exhibit super-hydrodynamic deviations from Nernst-Einstein predictions. Bridging this database analysis with microrheology and conductivity measurements, we validate the apparent superhydrodynamic ion transport in a common class of ionic liquids.

When compared to explanations that ascribe viscosity-conductivity scaling discrepancies to fractional ion dissociation, our findings would suggest over 100\% dissociation is achieved for some ionic liquids, and that increasing temperature drives ion association. This contradicts the Boltzmann population distribution which requires increases in thermal energy to drive dissociation. Both facts suggest alternative explanations 
for viscosity-conductivity scaling deviations are necessary, such as field-induced alterations to ionic liquid correlations and structuring, and its effect on super-hydrodynamic transport mechanisms. We conclude that Nernst-Einstein ratio does not directly quantify the fraction of free ions.

Looking to what features characterize the molar conductivity, the Stokes Einstein diffusion coefficients of each ion are the best predictors of the conductivity, consistent with the Nernst-Einstein model. Thus, the Nernst-Einstein model is likely the best sparse model that describes the coupling of conductivity and viscosity in ionic liquids. By including geometric features of ions in models, predictive performance can be improved, but the presence of both super-hydrodynamic and sub-hydrodynamic deviations remains. Using neural network models containing both experimental and computational features, we achieve improved conductivity prediction. Importantly, we show that ionic liquid molar conductivity can be predicted from computational features alone, enabling predictions of molar conductivity without experimental data.

Notably, many ionic liquids that show the greatest promise as electrolytes for lithium-ion batteries fall in the regime of super-hydrodynamic viscosity-conductivity scaling. Hence, our analysis suggests that superhydrodynamic transport may be a desired feature of ionic liquids and other correlated super-concentrated electrolytes. However, it will be important to explore how these scaling deviations translate to efficient transport of redox ions in multi-component electrolyte blends, such as lithium salt/ionic liquid blends.

\section{Experimental}

\section{Microrheology Measurements}

Ionic liquids were purchased from IoLiTec and purified in-house via a charcoal wash in solvent, followed by subsequent filtering, centrifugation, and drying for at least 48 hours in a vacuum oven at $100^{\circ} \mathrm{C}$. Silica tracer particles were added to each ionic liquid to achieve a dilute concentration of probes for tracking. Particles were either added as dry particles, or with solvent, in which case, the ionic liquids were dried again. Samples were sonicated in a bath sonicator for at least 15 minutes prior to any microrheological measurements. Average particle sizes were confirmed via scanning electron microscopy. All sample preparation was performed in a laminar flow hood to avoid contaminant particulates.

For microrheology measurements, a Nikon Eclipse Ti-2 microscope is connected to a Photometrics Prime 95B camera and fitted with a darkfield condenser to maximize signal-to-noise ratio in particle tracking of $686 \mathrm{~nm}$ silica nanospheres. A total of 60x magnification was used was used, comprised of a 40x objective lens and a $1.5 x$ magnification telescope. Pixel information was converted to spatial information using an automatic calibration with a motor-encoded stage. The trajectories of particles are recorded over four orders of magnitude of time ( $\sim 10^{-3} \mathrm{~s}$ to $\left.\sim 10 \mathrm{~s}\right)$ by separately recording short and long timescale experiments with framerates of $>450 \mathrm{fps}$ and $10 \mathrm{fps}$ respectively. Recordings are performed at least $100 \mu \mathrm{m}$ from channel 
edges to satisfy boundary conditions in Brownian models that are used to estimate viscosity. Temperature fluctuations are less than $0.2^{\circ} \mathrm{C}$ throughout the duration of experiments. Experiments were performed with about $30 \mu L$ of ionic liquid in grease-sealed, commercially available microfluidic channels (Ibidi) to ensure sample integrity as well as to eliminate convective flow due to evaporation or condensation. All reported values include at least 3 repetitions of each timescale. Raw image data is filtered with a bandpass filter to enhance signal-to-noise ratio. Images are then passed through a thresholding process to separate particles from the background. Particle trajectories are converted into mean-squared-displacement plots. Viscosities are then extracted from the resulting linear mean-squared-displacement plots via the Stokes-Einstein Equation and Brownian Dynamics in two dimensions:

$$
\begin{gathered}
M S D=\left\langle\Delta r_{i}^{2}\right\rangle=4 D_{\text {probe }} t_{\text {lag }} \\
D_{\text {probe }}=\frac{k T}{6 \pi \eta r_{\text {probe }}}
\end{gathered}
$$

Here, $t_{\text {lag }}$ is the lagtime, the duration of the particle trajectory in consideration, $M S D$ is the 2-dimensional mean squared displacement over the lagtime in consideration, and $D_{\text {probe }}$ is the Stokes-Einstein diffusivity of the tracer probe. Thus, the slope of the mean-squared displacement plot can be used to derive the viscosity of the sample if the particle size and temperature is known. A sample plot is shown in the SI, Figure S1. Viscosities of ionic liquids measured via microrheology in this study were always below $5.5 \%$ error of the values reported at the same temperature in the ILThermo database using macroscopic rheological techniques.

\section{Conductivity Measurements}

Ionic liquid samples for conductivity measurements were prepared in the same manner as the microrheology measurements without the silica addition. To model the relationship between temperature and conductivity of select ionic liquids, a Bio-Logic SAS SP-300 potentiostat was used to perform PotentioElectrochemical Impedance Spectroscopy (PEIS) with an amplitude of $10 \mathrm{mV}$ around the open circuit potential at frequencies from $10 \mathrm{~Hz}$ to $100 \mathrm{kHz}$. Three measurements were performed per frequency. 0.5 $\mathrm{mL}$ samples were temperature controlled in a dish of sand on a hotplate connected to an external temperature probe for temperatures of $\sim 30^{\circ} \mathrm{C}$ and above. A mercury thermometer was used to confirm temperatures. A custom two electrode conductivity probe was calibrated using triplicate measurements of $500 \mu \mathrm{S} / \mathrm{cm}, 5000 \mu \mathrm{S} / \mathrm{cm}$, and $12.88 \mathrm{mS} / \mathrm{cm} \mathrm{KCl}$ conductivity standards, and calibrations were validated each day new measurements were taken. Conductivity measurements for each ionic liquid were performed across five different temperatures with three repetitions at each temperature to enable estimation of the conductivity of ionic liquids as a function of temperature. Conductivity was extracted by fitting PEIS data 
to an equivalent circuit containing a resistor and constant phase element in series and extracting the resistive component. All measurements were performed inside a nitrogen glovebox with oxygen and water contents below $1 \mathrm{ppm}$.

\section{Associated Content}

The following files are available free of charge:

- Cashen, 2021. Supplementary Information (pdf)

- Decision Tree Regression Model - Normalized Features (svg)

- Interactive Nernst Einstein Plot (html)

- NE - High Slope Anions (png)

- NE - Medium Slope Anions (png)

- NE - Low Slope Anions (png)

- NE - High Slope Cations (png)

- NE - Medium Slope Cations (png)

- NE - Low Slope Cations (png)

\section{Corresponding Author}

* Matthew A. Gebbie - Department of Chemical and Biological Engineering, University of Wisconsin Madison, Madison, Wisconsin, 53706, United States; Email: gebbie@wisc.edu

\section{Author Contributions}

Research was designed by M.A.G. and R.K.C.. Experimental research was performed by R.K.C., A.J.S., and M.M.D.. Computational studies were performed by R.K.C.. Data was analyzed by R.K.C. and M.A.G.. This manuscript was written by R.K.C. and M.A.G..

\section{References}

1 Goodenough, J. B. \& Kim, Y. Challenges for Rechargeable Li Batteries. Chem. Mater. 22, 587-603, (2009).

2 Liu, Y., Zhu, Y. \& Cui, Y. Challenges and opportunities towards fast-charging battery materials. Nature Energy 4, 540-550, (2019).

3 Diederichsen, K. M., McShane, E. J. \& McCloskey, B. D. Promising Routes to a High $\mathrm{Li}+$ Transference Number Electrolyte for Lithium Ion Batteries. ACS Energy Letters 2, 2563-2575, (2017).

4 Horwitz, G., Rodríguez, C. R., Steinberg, P. Y., Burton, G. \& Corti, H. R. Mobilityviscosity decoupling and cation transport in water-in-salt lithium electrolytes. Electrochimica Acta 359, (2020).

5 Rakov, D. A. et al. Engineering high-energy-density sodium battery anodes for improved cycling with superconcentrated ionic-liquid electrolytes. Nat Mater 19, 1096-1101, (2020).

6 Yamada, Y. et al. Unusual stability of acetonitrile-based superconcentrated electrolytes for fast-charging lithium-ion batteries. J. Am. Chem. Soc. 136, 5039-5046, (2014).

7 Wang, J. et al. Superconcentrated electrolytes for a high-voltage lithium-ion battery. Nat Commun 7, 12032, (2016).

8 Borodin, O., Self, J., Persson, K. A., Wang, C. \& Xu, K. Uncharted Waters: SuperConcentrated Electrolytes. Joule 4, 69-100, (2020). 
Araque, J. C. \& Margulis, C. J. In an ionic liquid, high local friction is determined by the proximity to the charge network. J. Chem. Phys. 149, 144503, (2018).

10 Feng, G. et al. Free and Bound States of Ions in Ionic Liquids, Conductivity, and Underscreening Paradox. Physical Review X 9, (2019).

11 Yamada, Y., Wang, J., Ko, S., Watanabe, E. \& Yamada, A. Advances and issues in developing salt-concentrated battery electrolytes. Nature Energy 4, 269-280, (2019).

12 Janek, J. \& Zeier, W. G. A solid future for battery development. Nature Energy 1, (2016).

13 MacFarlane, D. R. et al. Ionic liquids and their solid-state analogues as materials for energy generation and storage. Nature Reviews Materials 1, (2016).

14 Welton, T. Ionic liquids: a brief history. Biophys. Rev. 10, 691-706, (2018).

15 Watanabe, M. et al. Application of Ionic Liquids to Energy Storage and Conversion Materials and Devices. Chem Rev 117, 7190-7239, (2017).

16 Hayes, R., Warr, G. G. \& Atkin, R. Structure and nanostructure in ionic liquids. Chem Rev 115, 6357-6426, (2015).

17 Arbizzani, C., Gabrielli, G. \& Mastragostino, M. Thermal stability and flammability of electrolytes for lithium-ion batteries. Journal of Power Sources 196, 4801-4805, (2011).

18 Welton, T. Room-temperature Ionic Liquids: Solvents for Synthesis and Catalysis. Chem. Rev. 99, 2071-2084, (1999).

19 Wasserscheid, P. \& Welton, T. Ionic Liquids in Synthesis. Vol. 1 (Wiley-Vch, 2003).

20 Armand, M., Endres, F., MacFarlane, D. R., Ohno, H. \& Scrosati, B. Ionic-liquid materials for the electrochemical challenges of the future. Nat Mater 8, 621-629, (2009).

21 Chen, F., Howlett, P. \& Forsyth, M. Na-Ion Solvation and High Transference Number in Superconcentrated Ionic Liquid Electrolytes: A Theoretical Approach. The Journal of Physical Chemistry C 122, 105-114, (2017).

22 Mendez-Morales, T. et al. Solvation of lithium salts in protic ionic liquids: a molecular dynamics study. J Phys Chem B 118, 761-770, (2014).

23 Nishio, K. et al. High Rate Capability of All-Solid-State Lithium Batteries Using QuasiSolid-State Electrolytes Containing Ionic Liquids. Journal of The Electrochemical Society 167, (2020).

24 Chagas, L. G., Jeong, S., Hasa, I. \& Passerini, S. Ionic Liquid-Based Electrolytes for Sodium-Ion Batteries: Tuning Properties To Enhance the Electrochemical Performance of Manganese-Based Layered Oxide Cathode. ACS Appl Mater Interfaces 11, 22278 22289, (2019).

25 Quan, P. et al. Safe sodium-ion battery using hybrid electrolytes of organic solvent/pyrrolidinium ionic liquid. Vietnam Journal of Chemistry 59, 17-26, (2021).

26 Lewandowski, A. \& Świderska-Mocek, A. Ionic liquids as electrolytes for Li-ion batteries-An overview of electrochemical studies. Journal of Power Sources 194, 601609, (2009).

27 Zhang, H. et al. Ionic liquid electrolyte with highly concentrated LiTFSI for lithium metal batteries. Electrochimica Acta 285, 78-85, (2018).

28 Forsyth, M. et al. Novel Na+ Ion Diffusion Mechanism in Mixed Organic-Inorganic Ionic Liquid Electrolyte Leading to High $\mathrm{Na}+$ Transference Number and Stable, High Rate Electrochemical Cycling of Sodium Cells. The Journal of Physical Chemistry C 120, 4276-4286, (2016). 
29 Monti, D., Jónsson, E., Palacín, M. R. \& Johansson, P. Ionic liquid based electrolytes for sodium-ion batteries: $\mathrm{Na}+$ solvation and ionic conductivity. Journal of Power Sources 245, 630-636, (2014).

30 Gao, X., Wu, F., Mariani, A. \& Passerini, S. Concentrated Ionic-Liquid-Based Electrolytes for High-Voltage Lithium Batteries with Improved Performance at Room Temperature. ChemSusChem 12, 4185-4193, (2019).

31 Osada, I., de Vries, H., Scrosati, B. \& Passerini, S. Ionic-Liquid-Based Polymer Electrolytes for Battery Applications. Angew Chem Int Ed Engl 55, 500-513, (2016).

32 Tokuda, H. et al. Physicochemical properties and structures of room-temperature ionic liquids. 3. Variation of cationic structures. Journal of Physical Chemistry B 110, 28332839, (2006).

33 Tokuda, H., Hayamizu, K., Ishii, K., Susan, M. A. B. H. \& Watanabe, M. Physicochemical properties and structures of room temperature ionic liquids. 2. Variation of alkyl chain length in imidazolium cation. Journal of Physical Chemistry B 109, 61036110, (2005).

34 Tokuda, H., Hayamizu, K., Ishii, K., Susan, M. A. B. H. \& Watanabe, M. Physicochemical properties and structures of room temperature ionic liquids. 1. Variation of anionic species. Journal of Physical Chemistry B 108, 16593-16600, (2004).

35 Araque, J. C., Yadav, S. K., Shadeck, M., Maroncelli, M. \& Margulis, C. J. How Is Diffusion of Neutral and Charged Tracers Related to the Structure and Dynamics of a Room-Temperature Ionic Liquid? Large Deviations from Stokes-Einstein Behavior Explained. J Phys Chem B 119, 7015-7029, (2015).

36 Dokko, K. et al. Direct Evidence for Li Ion Hopping Conduction in Highly Concentrated Sulfolane-Based Liquid Electrolytes. J Phys Chem B 122, 10736-10745, (2018).

$37 \mathrm{Xu}, \mathrm{W} .$, Cooper, E. I. \& Angell, C. A. Ionic liquids: Ion mobilities, glass temperatures, and fragilities. Journal of Physical Chemistry B 107, 6170-6178, (2003).

38 Videa, M. \& Angell, C. A. Glass formation, ionic conductivity, and conductivity/viscosity decoupling, in LiAlCl4 + LiClO4 and $\mathrm{LiAlCl} 4+\mathrm{LiAlCl} 3 \cdot$ imide solutions. Journal of Physical Chemistry B 103, 4185-4190, (1999).

39 Schreiner, C., Zugmann, S., Hartl, R. \& Gores, H. J. Fractional walden rule for ionic liquids: Examples from recent measurements and a critique of the so-called ideal $\mathrm{KCl}$ line for the walden plot. Journal of Chemical and Engineering Data 55, 1784-1788, (2010).

40 Xiao, W., Yang, Q. \& Zhu, S. Comparing ion transport in ionic liquids and polymerized ionic liquids. Sci. Rep. 10, 7825, (2020).

41 Ichikawa, T., Kato, T. \& Ohno, H. Dimension control of ionic liquids. Chem. Commun. (Camb.) 55, 8205-8214, (2019).

42 Lesnicki, D., Gao, C. Y., Limmer, D. T. \& Rotenberg, B. On the molecular correlations that result in field-dependent conductivities in electrolyte solutions. ArXiv, 1-13, (2021).

43 Nordness, O. \& Brennecke, J. F. Ion Dissociation in Ionic Liquids and Ionic Liquid Solutions. Chem Rev 120, 12873-12902, (2020).

44 MacFarlane, D. R. et al. On the concept of ionicity in ionic liquids. Phys. Chem. Chem. Phys. 11, 4962-4967, (2009).

45 Every, H. A., Bishop, A. G., MacFarlane, D. R., Orädd, G. \& Forsyth, M. Transport properties in a family of dialkylimidazolium ionic liquids. Phys. Chem. Chem. Phys. 6, 1758-1765, (2004). 
46 Noda, A., Hayamizu, K. \& Watanabe, M. Pulsed-gradient spin-echo 1H and 19F NMR ionic diffusion coefficient, viscosity, and ionic conductivity of non-chloroaluminate room-temperature ionic liquids. Journal of Physical Chemistry B 105, 4603-4610, (2001).

47 Dong, Q. et al. ILThermo: A Free-Access Web Database for Thermodynamic Properties of Ionic Liquids. J. Chem. Eng. Data 52, 1151-1159, (2007).

48 Kazakov, A. et al. (ed National Institute of Standards and Technology) (National Institute of Standards and Technology, National Institute of Standards and Technology, 2015).

49 Kim, S. et al. PubChem in 2021: new data content and improved web interfaces. Nucleic Acids Research 49, 1388-1395, (2020).

50 Bolton, E. E. et al. PubChem3D: A new resource for scientists. J. Cheminform. 3, 1-15, (2011).

51 Landrum, G. (Https://Www.Rdkit.Org/, 2019).

52 Mariani, A. et al. The unseen evidence of Reduced Ionicity: The elephant in (the) room temperature ionic liquids. J. Mol. Liq. 324, (2021).

53 Zarrougui, R., Raouafi, N. \& Lemordant, D. New Series of Green Cyclic AmmoniumBased Room Temperature Ionic Liquids with Alkylphosphite-Containing Anion: Synthesis and Physicochemical Characterization. J. Chem. Eng. Data 59, 1193-1201, (2014).

54 Rose, K. A. et al. Particle tracking of nanoparticles in soft matter. Journal of Applied Physics 127, (2020).

55 Dear, R. D., Worrall, E. K., Gault, W. D. \& Ritchie, G. A. Microrheological investigations in ionic liquids using optical trapping techniques. J Phys Chem B 117, 10567-10571, (2013).

56 Matsumoto, A., Del Giudice, F., Rotrattanadumrong, R. \& Shen, A. Q. Rheological Scaling of Ionic-Liquid-Based Polyelectrolytes in Ionic Liquid Solutions. Macromolecules 52, 2759-2771, (2019).

57 Koutsoukos, S., Philippi, F., Malaret, F. \& Welton, T. A review on machine learning algorithms for the ionic liquid chemical space. Chem. Sci. 12, 6820-6843, (2021).

58 Tokuda, H., Tsuzuki, S., Susan, M. A. B. H., Hayamizu, K. \& Watanabe, M. How ionic are room-temperature ionic liquids? An indicator of the physicochemical properties. Journal of Physical Chemistry B 110, 19593-19600, (2006).

59 Gebbie, M. A., Dobbs, H. A., Valtiner, M. \& Israelachvili, J. N. Long-range electrostatic screening in ionic liquids. Proc. Natl. Acad. Sci. U. S. A. 112, 7432-7437, (2015).

60 Gebbie, M. A. et al. Long range electrostatic forces in ionic liquids. Chem. Commun. (Camb.) 53, 1214-1224, (2017).

61 Abbott, A. P. Model for the conductivity of ionic liquids based on an infinite dilution of holes. Chemphyschem 6, 2502-2505, (2005).

62 McEwen, A. B., Ngo, H. L., LeCompte, K. \& Goldman, J. L. Electrochemical Properties of Imidazolium Salt Electrolytes for Electrochemical Capacitor Applications. Journal of The Electrochemical Society 146, 1687-1695, (1999).

63 Marcus, Y. Are Ionic Stokes Radii of Any Use? Journal of Solution Chemistry 41, 20822090, (2012).

$64 \mathrm{Xu}, \mathrm{W}$. \& Angell, C. A. Solvent-Free Electrolytes with Aqueous Solution-Like Conductivities. Science 302, 422-425, (2003). 
65 Hollóczki, O., Malberg, F., Welton, T. \& Kirchner, B. On the origin of ionicity in ionic liquids. Ion pairing versus charge transfer. Phys. Chem. Chem. Phys. 16, 16880-16890, (2014).

66 Driver, G. W. et al. Correlated/non-correlated ion dynamics of charge-neutral ion couples: the origin of ionicity in ionic liquids. Phys. Chem. Chem. Phys. 19, 4975-4988, (2017).

67 Harris, K. R. Can the Transport Properties of Molten Salts and Ionic Liquids Be Used To Determine Ion Association? J Phys Chem B 120, 12135-12147, (2016).

68 Liu, H. \& Maginn, E. An MD study of the applicability of the Walden rule and the Nernst-Einstein model for ionic liquids. Chemphyschem 13, 1701-1707, (2012).

69 Harris, K. R. Relations between the fractional stokes-einstein and Nernst-Einstein equations and velocity correlation coefficients in ionic liquids and molten salts. Journal of Physical Chemistry B 114, 9572-9577, (2010).

70 Kashyap, H. K., Annapureddy, H. V., Raineri, F. O. \& Margulis, C. J. How is charge transport different in ionic liquids and electrolyte solutions? J Phys Chem B 115, 1321213221, (2011).

71 Marcolongo, A. \& Marzari, N. Ionic correlations and failure of Nernst-Einstein relation in solid-state electrolytes. Physical Review Materials 1, (2017).

72 Angell, C. A., Ansari, Y. \& Zhao, Z. Ionic liquids: past, present and future. Faraday Discuss. 154, 9-27; discussion 81-96, 465-471, (2012).

73 Anouti, M., Caillon-Caravanier, M., Dridi, Y., Galiano, H. \& Lemordant, D. Synthesis and characterization of new pyrrolidinium based protic ionic liquids. Good and superionic liquids. Journal of Physical Chemistry B 112, 13335-13343, (2008).

74 Philippi, F. et al. Ether functionalisation, ion conformation and the optimisation of macroscopic properties in ionic liquids. Phys. Chem. Chem. Phys. 22, 23038-23056, (2020).

75 Wojnarowska, Z., Wang, Y., Paluch, K. J., Sokolov, A. P. \& Paluch, M. Observation of highly decoupled conductivity in protic ionic conductors. Phys. Chem. Chem. Phys. 16, 9123-9127, (2014).

76 Mogurampelly, S., Keith, J. R. \& Ganesan, V. Mechanisms Underlying Ion Transport in Polymerized Ionic Liquids. J. Am. Chem. Soc. 139, 9511-9514, (2017).

77 Yu, Z. et al. Asymmetric Composition of Ionic Aggregates and the Origin of High Correlated Transference Number in Water-in-Salt Electrolytes. J. Phys. Chem. Lett. 11, 1276-1281, (2020).

78 Sangoro, J. R., Iacob, C., Serghei, A., Friedrich, C. \& Kremer, F. Universal scaling of charge transport in glass-forming ionic liquids. Phys. Chem. Chem. Phys. 11, 913-916, (2009).

79 Ugata, Y. et al. Li-ion hopping conduction in highly concentrated lithium bis(fluorosulfonyl)amide/dinitrile liquid electrolytes. Phys. Chem. Chem. Phys. 21, 97599768, (2019).

80 Nurnberg, P. et al. Superionicity in Ionic-Liquid-Based Electrolytes Induced by Positive Ion-Ion Correlations. J. Am. Chem. Soc., (2022). 